\title{
Unified set of atomic transition probabilities for neutral argon
}

\author{
W. L. Wiese \\ National Institute of Standards and Technology, Gaithersburg, Maryland 20899
}

J. W. Brault

National Solar Observatory, Box 26732, Tucson, Arizona 85726-6732

K. Danzmann

Department of Physics, Stanford University, Stanford, California 94305-2196

V. Helbig

Institut für Experimentalphysik, Universität Kiel, 2300 Kiel, Federal Republic of Germany

M. Kock

Institut für Plasmaphysik, Universität Hannover, 3000 Hannover 1, Federal Republic of Germany

(Received 2 September 1988)

\begin{abstract}
The atomic transition probabilities and radiative lifetimes of neutral argon have been the subject of numerous experiments and calculations, but the results exhibit many discrepancies and inconsistencies. We present a unified set of atomic transition probabilities, which is consistent with essentially all recent results, albeit sometimes only after critical reanalysis. The data consistency and scale confirmation has been achieved in two ways. (i) We have carried out some lifetime-branching-ratio measurements for a principal $5 p$ level and the associated $4 s-5 p$ transitions. These measurements have very closely confirmed the accuracy of the results of recent independent emission experiments. (ii) We have critically reanalyzed and revised the literature data for the $4 s-4 p$ transitions, as well as utilized the results of a similar critical analysis for the $4 s-5 p$ transition array, to establish complete sets of absolute data for these arrays. We have found these data to be mutually consistent from cross-correlation checks between the two arrays, using recent literature data. Finally, we have proposed renormalization factors for other argon transitions based on this analysis.
\end{abstract}

\section{INTRODUCTION}

Argon, an inert atomic gas, has been one of the most attractive media for testing and applying the methods of plasma spectroscopy, including the determination of atomic transition probabilities for spontaneous emission. Also, argon has often been selected to test the various techniques to determine mean lifetimes of excited atomic states. During the last 30 years, at least 35 transition probability determinations utilizing emission spectroscopy $^{1-35}$ and 24 atomic lifetime determinations ${ }^{26,35-57}$ have been performed on neutral argon. When such a large number of experiments is involved, a certain amount of scatter will show up in the numerical results. But, surprisingly, the scatter-both in the emission and lifetime data-turned out to be much larger than expected, with many discrepancies outside the mutual error estimates. From the emission experiments, two scales for the atomic-transition-probability data seemed to evolve, ${ }^{58}$ which differed by about $30 \%$.

For the prominent $4 s-5 p$ lines, several recent experiments $24,27,28$ as well as critical evaluations of earlier work $^{27,59,60}$ have now produced a satisfactory understanding of the major causes of the discrepancies.
Corrections of some earlier work, suggested by these critical analyses, have produced consistency among most data. But all these data are based on the absoluteemission approach only. To achieve further progress it becomes necessary (a) to supplement this "emission" scale with an independent approach, (b) to extend the critical evaluations to other known lifetime and transition-probability data of argon, especially to the important $4 s-4 p$ array, and (c) to make an attempt to assemble all these data into one unified comprehensive set. This is the main objective of this paper, and to this end we have undertaken some required lifetime and branching-ratio measurements.

\section{APPROACH}

This paper has two main parts. (a) We describe an experiment comprising lifetime measurements of an Ar I 5p level as well as extensive branching ratio measurements for all transitions originating from this level. (b) We critically review the lifetime and transition probability data for the two most important groups of Ar I lines-the lines of the $4 s-4 p$ and $4 s-5 p$ transition arrays-and propose a unified absolute data set, which is shown to be consistent with the large majority of recent results.

(c) 1989 The American Physical Society 


\section{A. Combined lifetime and branching-ratio measurements of some $4 s-5 p$ transitions}

The combination of lifetime and branching-ratio measurements is an attractive technique for the accurate determination of atomic transition probabilities. But for the Ar I $5 p$ levels, this approach is difficult to carry out because (a) numerous emission-line branches, typically about 15, go from each of the ten $5 p$ levels into the lower $3 d, 5 s$, and $4 s$ levels (see Fig. 1), with a few lines located in the optical range and the large majority being infrared transitions, and (b) the lines of main interest, the $4 s-5 p$ transitions in the blue-green range of the visible spectrum, are relatively weak within this set of lines. Thus very accurate measurements of all these branches in widely different regions of the spectrum are requiredwhich is probably the reason why no such measurements exist as yet.

1. Experimental approach. We have selected the $5 p\left[\frac{5}{2}\right]_{2}$ level $\left(3 p_{8}\right.$ in Paschen notation) and all its radiative branches for our measurements. A wavelength range from 400 to $6100 \mathrm{~nm}$ had to be covered with high resolution to measure all 16 possible branches from this level. We found the $1-\mathrm{m}$ Fourier transform spectrometer ${ }^{61}$ at the National Solar Observatory, Tucson, Arizona to be well suited for this task. A folded Michelson configuration is employed and the total internal path length of $12 \mathrm{~m}$ is completely evacuated during operation. The maximum path-length difference is half a meter, if interferograms symmetric about the zeroth-order fringe are to be obtained. One of the mirrors can be offset to yield a maximum path-length difference of $1 \mathrm{~m}$ for onesided interferograms. During one scan of the mirrors a

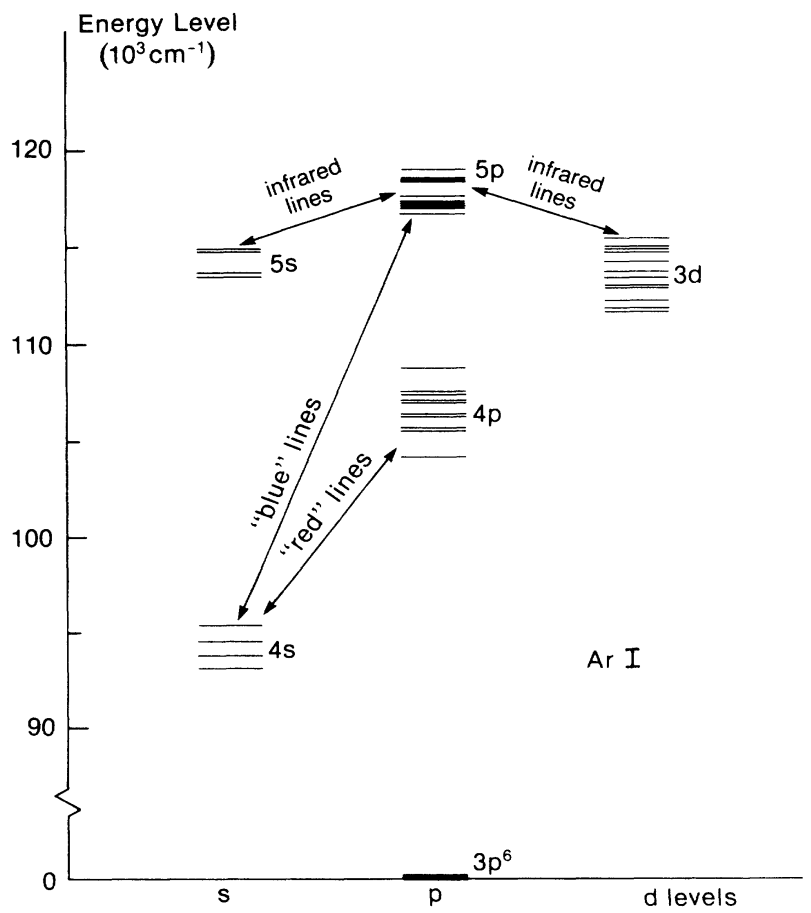

FIG. 1. Partial energy-level diagram for Ar I. maximum of one million data points are sampled at a typical rate of $2.5 \mathrm{kHz}$. Two outputs with complementary phases are provided and two detectors with different sensitivity may be used simultaneously to extend the useful wavelength interval of a single scan. A total wavelength range from 240 to $18000 \mathrm{~nm}$ is accessible in our overlapping segments depending on the type of beam splitters and optics installed. Because of these boundary conditions our measurements had to be split into three runs.

The first run served to bridge the large gap between the blue lines and the first group of infrared lines at $2512 \mathrm{~nm}$ and below. This run covered the range from 400 to 2600 $\mathrm{nm}$, using Ultrasil beam splitters with aluminum coating.

We recorded the ir lines with an indium-antimonide detector and simultaneously the blue line at $430 \mathrm{~nm}$ with a silicon $p-i-n$ diode. The blue part of the spectrum was undersampled and recorded in third order to achieve a resolution of $0.011 \mathrm{~cm}^{-1}$. No apodization procedure was employed during the Fourier transformation. To avoid overlapping of the aliases of the blue part with the rest of the spectrum we had to insert appropriate filters to restrict the spectral response of the instrument to narrow bands around the lines of interest. A Schott 430 filter was used for the blue channel and a wedged germanium filter for the infrared channel. An additional advantage of restricting the spectral response was a dramatic reduction in overall noise as compared to broadband spectra. In Fourier spectra the overall noise is determined by the shot noise in the strongest lines, and the strong red argon lines were eliminated in our case.

As a light source we used a high-current hollow cathode as described by Danzmann and Kock. ${ }^{62}$ This light source is especially suitable for measurements requiring long integration times because the intensities of spectral lines produced by this source have proven to be exceptionally stable in time. It should be emphasized, however, that all spectral lines were recorded simultaneously. Any temporal drift in the discharge conditions does not change the measured relative line intensities.

We used a nickel cathode with a bore of $8 \mathrm{~mm}$ diameter and $100 \mathrm{~mm}$ length. The discharge was operated in spectroscopically pure argon at a presure of $130 \mathrm{~Pa}$ (1 Torr). Three spectra were taken at discharge currents of $1,1.5$, and 2 A. Each spectrum was obtained by adding together four consecutive scans.

A second run covered the spectral range from 300 to $550 \mathrm{~nm}$ to further improve the relative intensity measurements of the three blue lines. The experimental conditions were almost identical to the first run, but we used two $p-i$ - $n$ diodes as detectors and restricted the spectral response by a copper sulfate filter. The unapodized resolution was set to $0.047 \mathrm{~cm}^{-1}$.

The third run covered the $1800 \mathrm{~nm}$ to (approximately) $6000 \mathrm{~nm}$ range to establish the relative intensities among the infrared lines. During this run we installed calcium fluoride beam splitters with $\mathrm{GaP}$ coating and used two indium antimonide detectors. Wavelengths shorter than $1800 \mathrm{~nm}$ were cut off by a germanium filter. The unapodized resolution was set to $0.009 \mathrm{~cm}^{-1}$.

To prevent atmospheric absorption by strong $\mathrm{CO}_{2}$ and 
$\mathrm{H}_{2} \mathrm{O}$ bands, the total external optical path was shielded by beam tubes and purged with dry nitrogen. The light source for this run was a water-cooled, medium-current hollow cathode with a vanadium cathode of $30 \mathrm{~mm}$ length and a bore of $6 \mathrm{~mm}$.

Three spectra were taken at discharge currents of 0.2 , 0.4 , and $0.56 \mathrm{~A}$ using an argon pressure of $130 \mathrm{~Pa}$ (1 Torr). Each spectrum was obtained by adding together eight consecutive scans.

To determine the spectral efficiency of the spectrometer, we recorded two reference spectra of radiation standards immediately before and after each run. We used a tungsten-ribbon lamp with a sapphire window and a tungsten-ribbon lamp with a fused-silica window, both of which were calibrated traceable to the National Bureau of Standards. By flipping a mirror the standard spectra could be recorded in the same optical setup as the argon spectra.

The resolution of the infrared reference spectra was set to $0.102 \mathrm{~cm}^{-1}$ to be able to resolve any residual atmospheric absorption bands, but no such bands could be detected. The resolution of the other reference spectra was set to $0.5 \mathrm{~cm}^{-1}$.

Since the radiation standards were calibrated at discrete wavelengths, we fitted blackbody curves to the supplied calibration tables in the relevant wavelength regions. The efficiency of the spectrometer was obtained by comparing the measured reference intensities at the line positions to the standard intensities calculated from the blackbody fit. The agreement between relative spectral efficiencies using the two different tungsten-ribbon lamps was better than $\pm 2.7 \%$ over the whole wavelength range.

In a separate experiment, the lifetime of the $5 p\left[\frac{5}{2}\right]_{2}$ level was determined by the method of laser-induced fluorescence (LIF). ${ }^{56}$ In order to apply this technique of selective level excitation to the highly excited Ar I $5 p$ level (14.5 eV above ground state, see Fig. 1), a low pressure argon plasma was generated in a specially designed dc hollow cathode discharge. Metastable argon atoms in the $4 s$ states were preferentially produced by extracting a beam from a hole at the end of the discharge into a vacuum chamber. (A detailed description of the source is found in Ref. 63.) Two of the four $4 s$ states are metastable, i.e., no electric dipole transitions are allowed to the ground state. Electrons in the metastable $4 s\left[\frac{3}{2}\right]_{2}^{o}$ state were thus resonantly excited into the $5 p\left[\frac{5}{2}\right]_{2}$ level with a tuned, nitrogen-pumped pulsed dye laser, and the spontaneous emission from the $5 p$ level was studied with the delayed coincidence method, applying a single-photon counting technique. The distribution of spontaneously emitted photons was accumulated and processed with a computer, based on about 8000 to 10000 photon counts for each decay. Further details of our experimental method and instrumentation are given in Refs. 56 and 57, where also possible alignment effects and the influence of weak magnetic fields are discussed.

2. Results. All of the 16 possible transitions from the $5 p\left[\frac{5}{2}\right]_{2}$ level could be measured except for the very weak 6123.9-nm line. For this line, which is about at the cutoff of our detector, we can only give a rough upper limit.

The line intensities $I_{k}$ were determined from the
Fourier spectra by calculating the areas under the line profiles over an interval of ten times the full width at half maximum. The line shapes were well resolved on our spectra. The profiles can be reproduced by almost pure Doppler broadening corresponding to kinetic temperatures of about 1400 to $2300 \mathrm{~K}$, depending on discharge current. No line-wing corrections had to be applied, because only relative intensity measurements of similar profiles out to the same cutoff points in the wings are involved.

We have checked the lines for self-absorption by comparing the results obtained at different discharge currents. No systematic variation could be found.

A critical examination of the line profiles did not reveal any signs of self-reversal in cool boundary layers. None of the lines of interest was disturbed by blends with other lines.

The branching ratio of a line is defined as the ratio of the line intensity (in terms of photons/s) to the sum of the intensities of all lines from that particular upper level. We have used the intensity $I_{0}$ of the line at $2512.5 \mathrm{~nm}$ as a reference to evaluate, in a first step, relative intensities

$$
L_{k}=I_{k} / I_{0}
$$

from three separate runs. From these relative intensities we subsequently calculated branching ratios

$$
R_{k}=L_{k} / \sum_{k} L_{k}
$$

The branching ratios of all 16 transitions from the Ar I level $5 p\left[\frac{5}{2}\right]_{2}$ are listed in Table I. The quoted uncertainties for each branch $k$ are two standard deviations of the mean values and have been obtained from the square root of the sum of the squares of two contributions: (i) The statistical uncertainty $\Delta L_{k} / L_{k}$ (fractional standard deviation of the relative intensity $L_{k}$ ), (ii) The uncertainty of the sum of all branches $S=\sum_{k} L_{k}$ as calculated from the fractional standard deviation

$$
\Delta S / S=\left(\sum_{k}\left(\Delta L_{k} / S\right)^{2}\right)^{1 / 2} .
$$

The lifetime of the $5 p\left[\frac{5}{2}\right]_{2}$ level was measured over a pressure range from $0.026 \mathrm{~Pa}\left(2 \times 10^{-4}\right.$ Torr $)$ to $0.26 \mathrm{~Pa}$ $\left(2 \times 10^{-3}\right.$ Torr $)$ in the observation area. A systematic dependence of the lifetime on pressure was observed, which is expected on account of collisional shortening of the lifetime due to the large deexcitation cross sections for the Ar I $5 p$ levels. (These are roughly estimated to be of the order of $20 \mathrm{~nm}^{2}$ for our experimental conditions, and are derived by assuming a thermal velocity distribution. ${ }^{56}$ ) Thus, a linear extrapolation to zero pressure was carried out. Our end result was a radiative lifetime of $\tau=152 \pm 7 \mathrm{~ns}$ for this argon level. During our work, we became aware of another very recent experiment by Hirabayashi et al. ${ }^{35}$ performed with the same laser-induced fluorescence technique, which produced a lifetime of $127 \pm 10 \mathrm{~ns}$, appreciably outside the mutual error limits. It appears that for the pressure range of their experiment, which is roughly a factor of $10^{3}$ higher than ours, the rather extended extrapolation to zero pressure is more uncertain than was estimated by the authors. Stryla 
et al. ${ }^{57}$ have now redetermined the lifetime of the $5 p\left[\frac{5}{2}\right]_{2}$ level with our LIF apparatus at even lower densities and obtained a value of $154 \pm 7 \mathrm{~ns}$. The arithmetic mean of the two closely agreeing results, $\tau=153 \mathrm{~nm}$, was therefore adopted for this work. An overall uncertainty of \pm 7 ns was obtained by combining the statistical measurement errors (two standard deviations) with an allowance for systematic errors.

\section{B. Critical review and data assembly}

In the spectrum of neutral argon, the two transition arrays $4 s-4 p$ and $4 s-5 p$-with a total of 30 transitions in each case-are of key importance, and give rise to the prominent "red" and "blue" groups of lines. This is seen in the partial energy-level diagram of Fig. 1.

1. $4 s-4 p$ transition array. In this case lifetime measurements of the $4 p$ levels are very attractive, since from a given $4 p$ level, radiative decays are only allowed to the four $4 s$ levels. Depending on the selection rules, one to four transitions (branches) are possible. Thus combinations of lifetime and emission branching-ratio measurements should produce accurate transition probability data for the $4 s-4 p$ lines. Lifetime measurements have recently undergone a considerable advance with the advent of the laser-induced-fluorescence technique. This laserbased selective excitation scheme, which totally eliminates the cascading problem, has been applied to all ten argon $4 p$ levels by Quadfasel and Helbig. ${ }^{55}$ Another very similar lifetime experiment by Chang and Setser, ${ }^{26}$ also with the selective tunable laser excitation technique, but on a slightly less comprehensive scale, is in excellent agreement with Quadfasel and Helbig's work, as shown in Table II. A lifetime measurement by Fujimoto et al. ${ }^{52}$ specifically for the $2 p_{4}$ level, again performed with the laser excitation technique, is also in excellent agreement with the other lifetime data (Fujimoto et al., ${ }^{52} 30.5 \mathrm{~ns}$; Quadfasel and Helbig, ${ }^{55} 29.3$ ns; Chang and Setser, ${ }^{26} 33.2$ ns).

Lifetime values and ratios of lifetimes from different levels may be also derived from comprehensive emission experiments. These data should be quite accurate on a relative basis, but less so on an absolute scale. Thus Table II contains also the (relative) results of two emission studies, ${ }^{10,33}$ which have been for purposes of easy comparison renormalized by a least-squares fit to the results of Ref. 55. It is seen that these relative results show the same distribution among the various levels as the directly obtained lifetimes.

Strong independent support for the absolute scale comes also from theory. Several calculations ${ }^{64-68}$ have been carried out for the $4 s-4 p$ array, with a very similar overall approach. While there are appreciable differences in the results for individual lines due to uncertainties in the determination of intermediate-coupling parameters, the overall theoretical scales are in close agreement with each other and in excellent agreement with the above recommended experimental scale. This is readily apparent from the data comparison between the lifetimes $\tau$ and the inverse transition probability sums $\left(\sum_{i} A_{k i}\right)^{-1}=\tau_{k}$ assembled in Table II. The table contains the theoretical data of Lilly ${ }^{64}$ as probably the most

TABLE I. Branching ratios $R$ of transitions from the Ar I $5 p\left[\frac{5}{2}\right]_{2}$ level $\left(E_{k}=116999.39 \mathrm{~cm}^{-1}\right)$ and corresponding transition probabilities, normalized to the average (153 ns) of the atomic lifetimes obtained by Ralfs (Ref. 56) and Stryla et al. (Ref. 57).

\begin{tabular}{|c|c|c|c|c|c|}
\hline Lower level & $E_{i}\left(\mathrm{~cm}^{-1}\right)$ & $\lambda(\mathrm{nm})$ & $100 R$ & $\Delta R(\%)$ & $\begin{array}{l}\text { Transition } \\
\text { probability } \\
A\left(10^{6} \mathrm{~s}^{-1}\right)\end{array}$ \\
\hline $4 s\left[\frac{3}{2}\right]_{2}^{o}$ & 99143.80 & 419.071 & 4.29 & 8 & 0.280 \\
\hline $4 s\left[\frac{3}{2}\right]_{1}^{o}$ & 93750.64 & 430.010 & 5.82 & 7 & 0.380 \\
\hline $4 s^{\prime}\left[\frac{1}{2}\right]_{1}^{o}$ & 95399.87 & 462.844 & 0.606 & 8 & 0.0396 \\
\hline $3 d\left[\frac{1}{2}\right]_{1}^{o}$ & 111818.09 & 1929.491 & 0.193 & 7 & 0.0126 \\
\hline $3 d\left[\frac{3}{2}\right]_{2}^{o}$ & 112138.98 & 2056.879 & 1.93 & 4 & 0.126 \\
\hline $3 d\left[\frac{7}{2}\right]_{3}^{o}$ & 113020.39 & 2512.509 & 21.25 & 3 & 1.39 \\
\hline $3 d\left[\frac{5}{2}\right]_{2}^{o}$ & 113426.05 & 2797.740 & 5.55 & 4 & 0.362 \\
\hline $5 s\left[\frac{5}{2}\right]_{2}^{o}$ & 113468.55 & 2831.415 & 13.88 & 5 & 0.907 \\
\hline $5 s\left[\frac{3}{2}\right]_{1}^{o}$ & 113643.26 & 2978.811 & 43.31 & 8 & 2.83 \\
\hline $3 d\left[\frac{5}{2}\right]_{3}^{o}$ & 114716.61 & 3045.369 & 2.25 & 10 & 0.148 \\
\hline $3 d\left[\frac{3}{2}\right]_{1}^{o}$ & 114147.75 & 3505.799 & 0.0623 & 4 & 0.00407 \\
\hline $3 d^{\prime}\left[\frac{5}{2}\right]_{2}^{o}$ & 114641.04 & 4239.098 & 0.455 & 5 & 0.0297 \\
\hline $3 d^{\prime}\left[\frac{3}{2}\right]_{2}^{o}$ & 114805.18 & 4556.209 & 0.189 & 5 & 0.0124 \\
\hline $3 d^{\prime}\left[\frac{5}{2}\right]_{3}^{o}$ & 114821.99 & 4591.384 & 0.198 & 6 & 0.0130 \\
\hline $5 s\left[\frac{1}{2}\right]_{1}^{o}$ & 114975.07 & 4938.586 & 0.0274 & 7 & 0.00179 \\
\hline $3 d^{\prime}\left[\frac{3}{2}\right]_{1}^{o}$ & 115366.90 & 6123.946 & $<0.06$ & & \\
\hline
\end{tabular}


TABLE II. Atomic lifetimes for the Ar I $4 p$ levels (in nanoseconds).

\begin{tabular}{|c|c|c|c|c|c|}
\hline \multirow[b]{2}{*}{ Level } & \multicolumn{2}{|c|}{ Direct lifetime measurements } & \multicolumn{2}{|c|}{ Emission measurements } & \multirow[b]{2}{*}{$\begin{array}{l}\text { Theory } \\
\text { Lilly }\end{array}$} \\
\hline & $\begin{array}{c}\text { Quadfasel } \\
\text { and Helbig }\end{array}$ & $\begin{array}{c}\text { Chang and } \\
\text { Setser }{ }^{b}\end{array}$ & $\begin{array}{c}\text { Shumaker } \\
\text { and Popenoe }\end{array}$ & $\begin{array}{l}\text { Musiol } \\
\text { et al. } \text {, } \mathrm{f}^{\mathrm{f}}\end{array}$ & \\
\hline $4 p^{\prime}\left[\frac{1}{2}\right]_{0}$ & 21.7 & & 23.0 & 21.5 & 22.6 \\
\hline $4 p^{\prime}\left[\frac{1}{2}\right]_{1}$ & 28.3 & 26.5 & 28.6 & 25.3 & 25.6 \\
\hline $4 p^{\prime}\left[\frac{3}{2}\right]_{2}$ & 29.0 & 27.6 & 29.4 & & 27.6 \\
\hline $4 p^{\prime}\left[\frac{3}{2}\right]_{1}$ & 29.3 & 33.2 & 31.3 & 30.6 & 29.0 \\
\hline $4 p\left[\frac{1}{2}\right]_{0}$ & 24.4 & & 25.5 & 24.8 & 22.8 \\
\hline $4 p\left[\frac{3}{2}\right]_{2}$ & 29.4 & 27.0 & 28.8 & & 27.0 \\
\hline $4 p\left[\frac{3}{2}\right]_{1}$ & 30.2 & 29.8 & 29.3 & & 28.7 \\
\hline $4 p^{\prime}\left[\frac{5}{2}\right]_{2}$ & 30.6 & 32.8 & 31.6 & 33.4 & 31.4 \\
\hline $4 p\left[\frac{5}{2}\right]_{3}$ & 30.7 & 30.1 & 29.9 & & 28.7 \\
\hline $4 p\left[\frac{1}{2}\right]_{1}$ & 40.5 & 42.2 & 38.2 & & \\
\hline
\end{tabular}

${ }^{\mathrm{a}}$ Reference 55 .

${ }^{\mathrm{b}}$ Reference 26.

${ }^{\mathrm{c}}$ Renormalized by a factor 0.85 for least-squares fit with Ref. 55 .

${ }^{\mathrm{d}}$ Reference 10.

${ }^{\text {e}}$ Relative data, normalized for best fit with Ref. 55 .

${ }^{\text {f }}$ Reference 33.

${ }^{\text {g}}$ Reference 64.

advanced theoretical result; however, as was just mentioned, earlier calculations by Garstang and Van Blerkom, ${ }^{65}$ Johnston, ${ }^{66}$ Gruzdev and Loginov, ${ }^{67}$ and Meiners ${ }^{68}$ produce quite similar results.

All the data in Table II show very similar patterns, and they clearly establish the lifetime data of Quadfasel and Helbig ${ }^{55}$ as the appropriate starting point for the absolute scale of the $4 s-4 p$ transition probabilities, which we thus adopt.

In order to proceed from this absolute scale to data for individual lines, we utilize (a) the renormalized comprehensive emission data by Shumaker and Popenoe $^{10}$ (see discussion below) as well as (b) branchingratio measurements. Among three available sets of branching ratios, ${ }^{26,29,31}$ we have selected those of Nick and Helbig ${ }^{31}$ as the most advanced and accurate set. Our main considerations for selecting their work were their detailed inclusion of line-wing intensity contributions, optical-depth studies and corrections, and their choice of the radiation standard. For the transitions originating from $2 p_{8}$ and $2 p_{10}$, which were not measured by Nick and Helbig, we use the branching ratios of Chang and Setser. ${ }^{26}$ Both data sets (a) and (b) have been normalized to the lifetime data of Quadfasel and Helbig ${ }^{55}$ which were adopted for our absolute scale. The agreement between the two data sets (a) and (b) is $7 \%$ or better, except for the $800.616-\mathrm{nm}$ line where it is $14 \%$. (For half of the 20 lines in common, the agreement is within $3 \%$.)

Another approach to determine the $4 s-4 p$ data is via absolute emission line intensities. Recent measurements with this method for a few selected lines were carried out by Nubbemeyer, ${ }^{23}$ and Nick and Helbig, ${ }^{31}$ and their results are shown in Table III. As was shown in a critical analysis of such argon emission experiments, ${ }^{59}$ these authors have addressed all critical factors, including the im- portant line-wing-intensity corrections, and the experiments are therefore expected to deliver accurate results. However, in Nubbemeyer's work ${ }^{23}$ the line-wing corrections have not been carried out as part of the line intensity measurement, but have only been considered as a correction factor in the final results. Thus his plasma diagnostic approach, done with untreated line intensity data, is not quite correct. An earlier emission experiment on the complete transition array by Shumaker and Popenoe ${ }^{10}$-with full corrections for the line wing intensities-suffers from the circumstance that the diagnostic technique used for the plasma analysis (Stark broadening) was at the time (1967) based on approximate theoretical data, which were repeatedly improved in the meantime. Thus Shumaker and Popenoe's relative scale should be excellent; their absolute scale is expected to be on the high side. Nick and Helbig ${ }^{31}$ have calculated that this scale should be changed by a factor of 0.78 based on the most accurate experimental Stark broadening results. We have therefore reduced Shumaker and Popenoe's absolute data by this factor.

In Table III, we have also compared these emission data $^{10,23,31}$ with results from the lifetime-branching-ratio approach. ${ }^{31,55}$ The numbers are consistent within the mutually estimated error limits. (It should be noted here that the emission scale correction by a factor of 0.78 for Shumaker and Popenoe's ${ }^{10}$ data, as mentioned above, is quite close to the correction factor of 0.85 cited in Table II, which we obtained from a least-squares fit of their inverse $A_{k i}$ sums to the lifetime data of Quadfasel and Helbig. ${ }^{55}$ )

Therefore, for the $4 s-4 p$ array of Ar I, very good overall consistency is found between the absolute-emission data, the lifetime-branching-ratio approach, and theory. One is thus in a position to assemble a data list for all indivi- 
TABLE III. Absolute transition probabilities for some prominent lines of the Ar I $4 s-4 p$ transition array (in $10^{5} \mathrm{~s}^{-1}$ ). QH, Quadfasel and Helbig (Ref. 55) (lifetime); NH, Nick and Helbig (Ref. 31) (branching ratios).

\begin{tabular}{cccccc}
\hline \hline Line & $\lambda(\mathrm{nm})$ & $\begin{array}{c}\text { Nick and } \\
\text { Helbig }^{\mathrm{a}}\end{array}$ & Nubbemeyer $^{\mathrm{b}}$ & $\begin{array}{c}\text { Emission method } \\
\text { Shumaker and } \\
\text { Popenoe, } \\
\text { renorm., }{ }^{\text {a c }}\end{array}$ & $\begin{array}{c}\text { Lifetime, } \\
\text { branching } \\
\text { ratios } \\
\text { QH }\end{array}$ \\
\hline $4 s\left[\frac{3}{2}\right]_{2}^{o}-4 p^{\prime}\left[\frac{3}{2}\right]_{1}$ & 714.704 & 5.75 & 6.51 & 5.52 & 6.51 \\
$4 s\left[\frac{3}{2}\right]_{2}^{o}-4 p^{\prime}\left[\frac{1}{2}\right]_{1}$ & 696.543 & 59 & & 56.9 & 66.0 \\
$4 s\left[\frac{3}{2}\right]_{1}^{o}-4 p^{\prime}\left[\frac{1}{2}\right]_{1}$ & 727.293 & 17 & 16.9 & 18.2 \\
$4 s\left[\frac{3}{2}\right]_{1}^{o}-4 p^{\prime}\left[\frac{1}{2}\right]_{0}$ & 667.728 & 2.19 & 2.03 & 2.46 \\
$4 s^{\prime}\left[\frac{1}{2}\right]_{1}^{o}-4 p^{\prime}\left[\frac{1}{2}\right]_{0}$ & 750.387 & 399 & 398 & 448 \\
\hline \hline
\end{tabular}

${ }^{a}$ Reference 31 .

${ }^{\mathrm{b}}$ Reference 23 .

${ }^{\mathrm{c}}$ Reference 10.

dual lines which should be, except for some weak lines, of high accuracy. The data we have selected are the averaged values of the emission measurements by Shumaker and Popenoe ${ }^{10}$ and the branching-ratio measurements by Nick and Helbig, ${ }^{31}$ which were both normalized to the lifetime data of Quadfasel and Helbig. ${ }^{55}$ The two data sets are in excellent agreement, exceeding a difference of $6 \%$ only for the $800.6-\mathrm{nm}$ line. For a few longerwavelength transitions, which were not measured by Nick and Helbig, we have utilized the branching ratios of Chang and Setzer ${ }^{26}$ which were also found to be in very good agreement with the data of Shumaker and Popenoe. The list of recommended data for the $4 s-4 p$ array is given in Table IV, with our accuracy estimates, which include the uncertainties given for the lifetime and averaged emission data.

2. $4 s-5 p$ transition array. For this group of 30 lines in the blue part of the spectrum, the data situation is quite different from the $4 s-4 p$ array. Much of this has to do with the accidental circumstance that these transitions are all rather weak. (a) Theoretical methods do not provide reliable results, because the transition integral for the $4 s-5 p$ array is very small. Its positive and negative parts nearly cancel each other, and thus make its precise determination very uncertain. ${ }^{68}$ (b) The combination technique of lifetime and branching-ratio measurements is much more difficult to apply to this transition array than to the $4 s-4 p$ array. This is due to the fact that each $5 p$ level has numerous radiative decay branches. Among these branches, the $5 p \rightarrow 4 s$ emission is relatively weak, and represents only a small fraction of the overall radiative decay rate (typically about $10 \%$, see Table I) so that very precise branching-ratio measurements are required in order to achieve acceptable accuracies. The large spectral range that must be covered presents significant technical difficulties, too. Our experiment described in Sec. II $\mathrm{A}$ is the first measurement of this kind. (c) $\mathrm{Be}$ cause of the difficulties encountered with approaches (a) and (b), the emission technique has been for a long time the main source of data for this array. Indeed, numerous emission experiments have been done for its various lines. This applies especially to the line at $430.010 \mathrm{~nm}$, which seems to be the generally preferred transition because it is a well isolated line. ${ }^{69}$ For this line, where 17 independent emission studies have been performed (Refs. 1-8, 12, 14, $16,18,20,23,24,27,28$, and 35$)$, the appearance of two

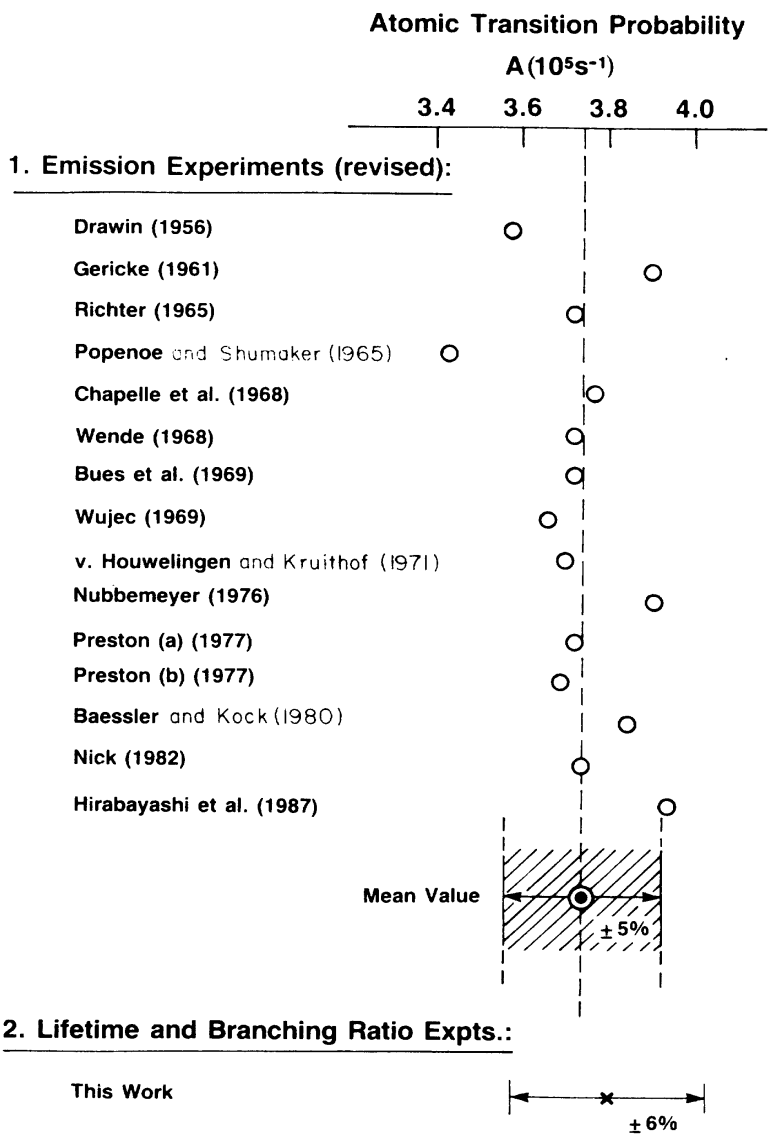

FIG. 2. Results of atomic-transition-probability measurements for the $4 s-5 p$ transition of Ar I at $430.010 \mathrm{~nm}$ : (1) emission experiments (Refs. 1-4, 12, 14, 16, 18, 20, 23, 24, 27, 28, and 35), critically analyzed and revised (Ref. 60); (2) lifetime-branching-ratio approach of this experiment. 
different scales for its transition probability, which are about $30 \%$ apart, was repeatedly noted. ${ }^{16,24,28,58} \mathrm{~A}$ recent detailed analysis ${ }^{60}$ has, however, reconciled the different data and has established one definite scale, which is consistent with all experiments after reinterpretation. Briefly summarized, this critical analysis has shown that several problem areas existed. (1) A plasma diagnostic problem arose in some early experiments ${ }^{1,3,18}$ due to the application of inadequate plasma linebroadening (Stark-broadening) data. These results have been corrected by the substitution of recent, more accurate Stark-broadening data. (2) Unrecognized deviations occurred from the assumed plasma model of local thermodynamic equilibrium (LTE). This problem again arose in early experiments, ${ }^{5-7}$ since no quantitative experimen- tal or theoretical LTE studies were available yet to serve as a reliable guide. In the meantime, a minimum value for the most critical parameter, the electron density in the plasmas, was established from several experimental and theoretical studies ${ }^{60}$ to be about $5 \times 10^{22} \mathrm{~m}^{-1}$, which thus represents the lower limit for the existence of LTE. Many experiments were performed at somewhat higher densities, up to $1.5 \times 10^{23} \mathrm{~m}^{-3}$, but some early experiments carried out at electron densities significantly below the critical value had to be excluded from final consideration. (3) The intensity contributions by the extended line wings were sometimes not included (Refs. 1, 2, 4, 12, 14, $16,18,20$, and 27$)$. This amounts to missing roughly $20 \%$ of the total line intensity and leads to an equal reduction of the transition probability. Therefore, such

TABLE IV. Recommended atomic transition probabilities for the Ar I $4 s-4 p$ transition array.

\begin{tabular}{|c|c|c|c|c|}
\hline \multicolumn{2}{|c|}{ Transition } & \multirow{2}{*}{$\frac{\lambda(\mathrm{nm})}{750.384}$} & \multirow{2}{*}{$\frac{A\left(10^{6} \mathrm{~s}^{-1}\right)}{44.5}$} & \multirow{2}{*}{$\begin{array}{c}\begin{array}{c}\text { Estimated } \\
\text { uncertainty }\end{array} \\
\pm 8 \%\end{array}$} \\
\hline $4 s^{\prime}\left[\frac{1}{2}\right]_{1}^{o}$ & $4 p^{\prime}\left[\frac{1}{2}\right]_{0}$ & & & \\
\hline & $4 p^{\prime}\left[\frac{1}{2}\right]_{1}$ & 826.452 & 15.3 & $\pm 5 \%$ \\
\hline & $4 p^{\prime}\left[\frac{3}{2}\right]_{2}$ & 840.821 & 22.3 & $\pm 5 \%$ \\
\hline & $4 p^{\prime}\left[\frac{3}{2}\right]_{1}$ & 852.144 & 13.9 & $\pm 8 \%$ \\
\hline & $4 p\left[\frac{1}{2}\right]_{0}$ & 857.806 & $<0.001$ & \\
\hline & $4 p\left[\frac{3}{2}\right]_{2}$ & 922.450 & 5.03 & $\pm 10 \%$ \\
\hline & $4 p\left[\frac{3}{2}\right]_{1}$ & 935.422 & 1.06 & $\pm 8 \%$ \\
\hline & $4 p^{\prime}\left[\frac{5}{2}\right]_{2}$ & 978.450 & 1.47 & $\pm 8 \%$ \\
\hline & $4 p\left[\frac{1}{2}\right]_{1}$ & 1148.811 & 0.19 & $\pm 30 \%$ \\
\hline \multirow[t]{4}{*}{$4 s^{\prime}\left[\frac{1}{2}\right]_{0}^{o}$} & $4 p^{\prime}\left[\frac{1}{2}\right]_{1}$ & 772.421 & 11.7 & $\pm 5 \%$ \\
\hline & $4 p^{\prime}\left[\frac{3}{2}\right]_{1}$ & 794.818 & 18.6 & $\pm 8 \%$ \\
\hline & $4 p\left[\frac{3}{2}\right]_{1}$ & 866.794 & 2.43 & $\pm 8 \%$ \\
\hline & $4 p\left[\frac{1}{2}\right]_{1}$ & 1047.005 & 0.98 & $\pm 15 \%$ \\
\hline \multirow[t]{9}{*}{$4 s\left[\frac{3}{2}\right]_{1}^{o}$} & $4 p^{\prime}\left[\frac{1}{2}\right]_{0}$ & 667.728 & 0.236 & $\pm 10 \%$ \\
\hline & $4 p^{\prime}\left[\frac{1}{2}\right]_{1}$ & 727.293 & 1.83 & $\pm 5 \%$ \\
\hline & $4 p^{\prime}\left[\frac{3}{2}\right]_{2}$ & 738.398 & 8.47 & $\pm 8 \%$ \\
\hline & $4 p^{\prime}\left[\frac{3}{2}\right]_{1}$ & 747.117 & 0.022 & $\pm 8 \%$ \\
\hline & $4 p\left[\frac{1}{2}\right]_{0}$ & 751.465 & 40.2 & $\pm 8 \%$ \\
\hline & $4 p\left[\frac{3}{2}\right]_{2}$ & 800.616 & 4.90 & $\pm 15 \%$ \\
\hline & $4 p\left[\frac{3}{2}\right]_{1}$ & 810.369 & 25.0 & $\pm 5 \%$ \\
\hline & $4 p^{\prime}\left[\frac{5}{2}\right]_{2}$ & 842.465 & 21.5 & $\pm 5 \%$ \\
\hline & $4 p\left[\frac{1}{2}\right]_{1}$ & 965.778 & 5.43 & $\pm 8 \%$ \\
\hline \multirow[t]{8}{*}{$4 s\left[\frac{3}{2}\right]_{2}^{o}$} & $4 p\left[\frac{1}{2}\right]_{1}$ & 696.543 & 6.39 & $\pm 5 \%$ \\
\hline & $4 p^{\prime}\left[\frac{3}{2}\right]_{2}$ & 706.722 & 3.80 & $\pm 8 \%$ \\
\hline & $4 p^{\prime}\left[\frac{3}{2}\right]_{1}$ & 714.704 & 0.625 & $\pm 8 \%$ \\
\hline & $4 p^{\prime}\left[\frac{3}{2}\right]_{2}$ & 763.511 & 24.5 & $\pm 8 \%$ \\
\hline & $4 p\left[\frac{3}{2}\right]_{1}$ & 772.376 & 5.18 & $\pm 5 \%$ \\
\hline & $4 p^{\prime}\left[\frac{5}{2}\right]_{2}$ & 801.479 & 9.28 & $\pm 8 \%$ \\
\hline & $4 p\left[\frac{5}{2}\right]_{3}$ & 811.531 & 33.1 & $\pm 8 \%$ \\
\hline & $4 p\left[\frac{1}{2}\right]_{1}$ & 912.297 & 18.9 & $\pm 8 \%$ \\
\hline
\end{tabular}


amounts have been added for all experiments where linewing corrections were not mentioned by the authors. (4) An inadequate definition of the emission layer occurred in one experiment, ${ }^{8}$ i.e., measurements were performed in a highly inhomogeneous plasma. These results were therefore excluded from further consideration.

The critical analysis ${ }^{60}$ finally established for the Ar I 430.010-nm line an atomic transition probability of
$A_{430.010}^{\prime}=3.74 \times 10^{5} \mathrm{~s}^{-1}$. This mean value of the revised results of 14 emission experiments agrees with all individual results within $\pm 5 \%$, as shown in Fig. 2, and it agrees almost exactly, i.e., within $\pm 1 \%$, with the recent precision experiments of Preston ${ }^{24}$ and Nick. ${ }^{28}$

The 430.010-nm line is, on the other hand, also one of the branches originating from the $5 p\left[\frac{5}{2}\right]_{2}$ level which we have determined in this experiment with our completely

TABLE V. Comparison of comprehensive emission data sets for the Ar I $4 s-5 p$ transition array, normalized to the earlier established value for the 430.010-nm line.

\begin{tabular}{|c|c|c|c|c|c|}
\hline & & & & ion probabilitie & $-1)$ \\
\hline & & $\lambda(\mathrm{nm})$ & Wende $^{\mathrm{a}}$ & $\begin{array}{l}\text { Bues, Haag, } \\
\text { and Richter }\end{array}$ & $\begin{array}{c}\text { Jones and } \\
\text { Wiese }^{c}\end{array}$ \\
\hline $4 s^{\prime}\left[\frac{1}{2}\right]_{1}^{o}$ & $5 p^{\prime}\left[\frac{1}{2}\right]_{0}$ & 425.936 & 3.9 & 4.04 & 3.99 \\
\hline & $5 p^{\prime}\left[\frac{1}{2}\right]_{1}$ & 433.534 & 0.35 & 0.376 & 0.436 \\
\hline & $5 p^{\prime}\left[\frac{3}{2}\right]_{2}$ & 433.356 & 0.59 & 0.553 & 0.562 \\
\hline & $5 p^{\prime}\left[\frac{3}{2}\right]_{1}$ & 434.517 & 0.27 & 0.314 & 0.308 \\
\hline & $5 p\left[\frac{1}{2}\right]_{0}$ & 451.073 & 1.2 & 1.14 & 1.20 \\
\hline & $5 p\left[\frac{3}{2}\right]_{2}$ & 458.929 & 0.0036 & 0.007 & 0.008 \\
\hline & $5 p\left[\frac{3}{2}\right]_{1}$ & 459.610 & 0.090 & 0.093 & 0.101 \\
\hline & $5 p^{\prime}\left[\frac{5}{2}\right]_{2}^{d}$ & 462.844 & 0.039 & 0.037 & 0.039 \\
\hline & $5 p\left[\frac{1}{2}\right]_{1}$ & 470.232 & 0.11 & 0.10 & 0.117 \\
\hline $4 s^{\prime}\left[\frac{1}{2}\right]_{0}^{o}$ & $5 p^{\prime}\left[\frac{1}{2}\right]_{1}$ & 418.188 & 0.56 & 0.529 & 0.593 \\
\hline & $5 p^{\prime}\left[\frac{3}{2}\right]_{1}$ & 419.103 & & 0.539 & \\
\hline & $5 p\left[\frac{3}{2}\right]_{1}$ & 442.400 & 0.0066 & 0.008 & \\
\hline & $5 p\left[\frac{1}{2}\right]_{1}$ & 452.232 & 0.091 & 0.091 & 0.0873 \\
\hline $4 s\left[\frac{3}{2}\right]_{1}^{o}$ & $5 p^{\prime}\left[\frac{1}{2}\right]_{0}$ & 397.972 & & & \\
\hline & $5 p^{\prime}\left[\frac{1}{2}\right]_{1}$ & 404.596 & 0.037 & 0.044 & \\
\hline & $5 p^{\prime}\left[\frac{3}{2}\right]_{2}$ & 404.442 & 0.34 & 0.310 & 0.349 \\
\hline & $5 p^{\prime}\left[\frac{3}{2}\right]_{1}$ & 405.453 & 0.023 & 0.028 & 0.0302 \\
\hline & $5 p\left[\frac{1}{2}\right]_{0}$ & 419.832 & 2.7 & 2.52 & 2.48 \\
\hline & $5 p\left[\frac{3}{2}\right]_{2}$ & 426.629 & 0.28 & 0.351 & 0.305 \\
\hline & $5 p\left[\frac{3}{2}\right]_{1}$ & 427.217 & 0.77 & 0.844 & 0.776 \\
\hline & $5 p^{\prime}\left[\frac{5}{2}\right]_{2}$ & 430.010 & 0.377 & 0.377 & 0.377 \\
\hline & $5 p\left[\frac{1}{2}\right]_{1}$ & 436.379 & 0.012 & 0.012 & \\
\hline $4 s\left[\frac{3}{2}\right]_{2}^{o}$ & $5 p\left[\frac{1}{2}\right]_{1}$ & 394.898 & 0.42 & 0.453 & 0.492 \\
\hline & $5 p^{\prime}\left[\frac{3}{2}\right]_{2}$ & 394.750 & 0.054 & 0.066 & 0.048 \\
\hline & $5 p^{\prime}\left[\frac{3}{2}\right]_{1}^{e}$ & 395.7 & & & \\
\hline & $5 p\left[\frac{3}{2}\right]_{2}$ & 415.859 & 1.3 & 1.40 & 1.50 \\
\hline & $5 p\left[\frac{3}{2}\right]_{1}$ & 416.418 & 0.27 & 0.312 & 0.281 \\
\hline & $5 p^{\prime}\left[\frac{5}{2}\right]_{2}{ }^{\mathrm{f}}$ & 419.071 & & 0.246 & \\
\hline & $5 p\left[\frac{5}{2}\right]_{3}$ & 420.067 & 1.00 & 0.939 & 0.961 \\
\hline & $5 p\left[\frac{1}{2}\right]_{1}$ & 425.118 & 0.092 & 0.123 & 0.117 \\
\hline
\end{tabular}

${ }^{\mathrm{a}}$ Reference 14

${ }^{\mathrm{b}}$ Reference 16.

${ }^{\mathrm{c}}$ Reference 34 .

${ }^{\mathrm{d}}$ The result of our lifetime-branching-ratio approach is $A=0.0383$ (see Table II).

${ }^{\mathrm{e}}$ Not observed.

${ }^{f}$ The result of our lifetime-branching-ratio approach is $A=0.280$ (see Table I). 
independent lifetime-branching-ratio approach. Our result, $A_{430.010}^{\prime \prime}=3.80 \times 10^{5} \mathrm{~s}^{-1} \pm 7 \%$ (see Table I), fully confirms the emission scale, from which it differs by only $1.6 \%$.

The absolute scale appears to be thus firmly settled, and we are now in a position to normalize, or renormalize, three fairly complete sets of emission data ${ }^{14,16,34}$ for the $4 s-5 p$ array to obtain numerical values for all individual lines. The emission-data sets were all obtained from photoelectric measurements with wall-stabilized arcs. Table V contains a comparison of these data sets, which are normalized to the $430.010-\mathrm{nm}$ line, and a generally very satisfactory agreement is seen. The only problem cases are (a) the nearly blended lines at 419.071 and $419.105 \mathrm{~nm}$, which have been separately measured only by Bues et al. ${ }^{16}$ (at lower densities), and (b) the weak lines at 458.929 and $442.400 \mathrm{~nm}$, where the available results differ appreciably.

We thus recommend for the lines of the $4 s-5 p$ array (a) to base the absolute scale on the arithmetic mean of (1) our result for the lifetime-branching-ratio approach and

TABLE VI. Recommended atomic transition probabilities for the Ar I $4 s-5 p$ transition array.

\begin{tabular}{|c|c|c|c|c|}
\hline \multicolumn{2}{|c|}{ Transition } & \multirow{2}{*}{$\frac{\lambda(\mathrm{nm})}{425.936}$} & \multirow{2}{*}{$\frac{A\left(10^{6} \mathrm{~s}^{-1}\right)}{3.98}$} & \multirow{2}{*}{$\begin{array}{c}\begin{array}{c}\text { Estimated } \\
\text { uncertainty }\end{array} \\
\pm 6 \%\end{array}$} \\
\hline $4 s^{\prime}\left[\frac{1}{2}\right]_{1}^{o}$ & $5 p^{\prime}\left[\frac{1}{2}\right]_{0}$ & & & \\
\hline & $5 p^{\prime}\left[\frac{1}{2}\right]_{1}$ & 433.534 & 0.387 & $\pm 12 \%$ \\
\hline & $5 p^{\prime}\left[\frac{3}{2}\right]_{2}$ & 433.356 & 0.568 & $\pm 7 \%$ \\
\hline & $5 p^{\prime}\left[\frac{3}{2}\right]_{1}$ & 434.517 & 0.297 & $\pm 10 \%$ \\
\hline & $5 p\left[\frac{1}{2}\right]_{0}$ & 451.073 & 1.18 & $\pm 7 \%$ \\
\hline & $5 p\left[\frac{3}{2}\right]_{2}$ & 458.929 & 0.0062 & $\pm 20 \%$ \\
\hline & $5 p\left[\frac{3}{2}\right]_{1}$ & 459.610 & 0.0947 & $\pm 9 \%$ \\
\hline & $5 p^{\prime}\left[\frac{5}{2}\right]_{2}$ & 462.844 & $0.0383^{\mathrm{a}}$ & $\pm 6 \%$ \\
\hline & $5 p\left[\frac{1}{2}\right]_{1}$ & 470.232 & 0.109 & $\pm 9 \%$ \\
\hline \multirow[t]{4}{*}{$4 s^{\prime}\left[\frac{1}{2}\right]_{0}^{o}$} & $5 p^{\prime}\left[\frac{1}{2}\right]_{1}$ & 418.188 & 0.561 & $\pm 9 \%$ \\
\hline & $5 p^{\prime}\left[\frac{3}{2}\right]_{1}$ & 419.103 & 0.539 & $\pm 15 \%$ \\
\hline & $5 p\left[\frac{3}{2}\right]_{1}$ & 442.400 & 0.0073 & $\pm 15 \%$ \\
\hline & $5 p\left[\frac{1}{2}\right]_{1}$ & 452.232 & 0.0898 & $\pm 8 \%$ \\
\hline \multirow[t]{9}{*}{$4 s\left[\frac{3}{2}\right]_{1}^{o}$} & $5 p^{\prime}\left[\frac{1}{2}\right]_{0}$ & 397.972 & & \\
\hline & $5 p^{\prime}\left[\frac{1}{2}\right]_{1}$ & 404.596 & 0.041 & $\pm 10 \%$ \\
\hline & $5 p^{\prime}\left[\frac{3}{2}\right]_{2}$ & 404.442 & 0.333 & $\pm 9 \%$ \\
\hline & $5 p^{\prime}\left[\frac{3}{2}\right]_{1}$ & 405.453 & 0.027 & $\pm 15 \%$ \\
\hline & $5 p\left[\frac{1}{2}\right]_{0}$ & 419.832 & 2.57 & $\pm 8 \%$ \\
\hline & $5 p\left[\frac{3}{2}\right]_{2}$ & 426.629 & 0.312 & $\pm 12 \%$ \\
\hline & $5 p\left[\frac{3}{2}\right]_{1}$ & 427.217 & 0.797 & $\pm 8 \%$ \\
\hline & $5 p^{\prime}\left[\frac{5}{2}\right]_{2}$ & 430.010 & 0.377 & $\pm 5 \%$ \\
\hline & $5 p\left[\frac{1}{2}\right]_{1}$ & 436.379 & 0.012 & $\pm 10 \%$ \\
\hline \multirow[t]{8}{*}{$4 s\left[\frac{3}{2}\right]_{2}^{o}$} & $5 p\left[\frac{1}{2}\right]_{1}$ & 394.898 & 0.455 & $\pm 9 \%$ \\
\hline & $5 p^{\prime}\left[\frac{3}{2}\right]_{2}$ & 394.750 & 0.056 & $\pm 15 \%$ \\
\hline & $5 p^{\prime}\left[\frac{3}{2}\right]_{1}^{\mathrm{b}}$ & 395.7 & & \\
\hline & $5 p\left[\frac{3}{2}\right]_{2}$ & 415.859 & 1.40 & $\pm 9 \%$ \\
\hline & $5 p\left[\frac{3}{2}\right]_{1}$ & 416.418 & 0.288 & $\pm 9 \%$ \\
\hline & $5 p^{\prime}\left[\frac{5}{2}\right]_{2}$ & 419.071 & $0.280^{c}$ & $\pm 8 \%$ \\
\hline & $5 p\left[\frac{5}{2}\right]_{3}$ & 420.067 & 0.967 & $\pm 7 \%$ \\
\hline & $5 p\left[\frac{1}{2}\right]_{1}$ & 425.118 & 0.111 & $\pm 15 \%$ \\
\hline
\end{tabular}

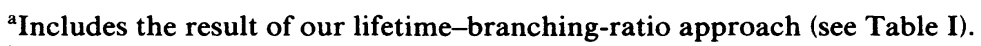

${ }^{\mathrm{b}}$ Line not observed.

${ }^{c}$ For this transition, which nearly overlaps with the line at $419.103 \mathrm{~nm}$, we apply the result of our lifetime-branching-ratio approach (see Table I) which is estimated to be much more accurate than the emission result of Bues et al. (Ref. 16). 
TABLE VII. Transition probability ratios for some $4 s-4 p$ and $4 s-5 p$ lines measured in emission.

\begin{tabular}{|c|c|c|c|c|c|}
\hline \multirow[b]{2}{*}{ Pair of transitions } & \multirow[b]{2}{*}{$\begin{array}{l}\text { Wavelengths } \\
(\mathrm{nm})\end{array}$} & \multirow[b]{2}{*}{$\begin{array}{l}\text { This } \\
\text { work }\end{array}$} & \multicolumn{3}{|c|}{ Transition probability ratios } \\
\hline & & & $\begin{array}{l}\text { Bues } \\
\text { et al. }{ }^{\text {a }}\end{array}$ & Nubbemeyer ${ }^{\mathrm{b}}$ & $\begin{array}{l}\text { van Houwelingen } \\
\text { and Kruithof }\end{array}$ \\
\hline $\begin{array}{l}4 s\left[\frac{3}{2}\right]_{2}^{o}-4 p^{\prime}\left[\frac{1}{2}\right]_{1} \\
4 s^{\prime}\left[\frac{1}{2}\right]_{1}^{o}-5 p^{\prime}\left[\frac{1}{2}\right]_{0}\end{array}$ & $696.5,425.9$ & 1.62 & & & 1.49 \\
\hline $\begin{array}{l}4 s\left[\frac{3}{2}\right]_{2}^{o}-4 p^{\prime}\left[\frac{3}{2}\right]_{1} \\
4 s\left[\frac{3}{2}\right]_{1}^{o}-5 p\left[\frac{5}{2}\right]_{2}\end{array}$ & $714.7,430.0$ & 1.68 & 1.46 & 1.66 & \\
\hline
\end{tabular}

${ }^{\text {a }}$ Reference 16.

${ }^{a}$ Reference 23 .

${ }^{\text {a }}$ Reference 20.

(2) the result of the emission experiment analysis, which is

$$
A_{430.010}=3.77 \times 10^{5} \mathrm{~s}^{-1} \text {; }
$$

and (b) to normalize the three emission-data sets discussed above $\mathrm{e}^{14,16,34}$ to this scale and to average them. This set of recommended data is given, with two standard deviations, in Table VI.

Finally, it is also essential that the two independently established data sets for the $4 s-4 p$ and $4 s-5 p$ arrays be consistent with each other. At this time, not many accurate cross connections between these transition arrays exist that could provide reliable tests. The available material, assembled in Table VII, indicates that consistency is achieved within the estimated error limits. The closest agreement is obtained with Nubbemeyer, ${ }^{23}$ whose precision data should carry considerable weight. But the data by Bues et al. ${ }^{16}$ are not very accurate (they estimate uncertainties of $\pm 20 \%$ ) so that the quoted comparison (as well as additional possible comparisons from their data set) are not a critical test.

\section{SUMMARY}

We have established a unified scale for the transition probabilities of Ar I by measuring some $4 s-5 p$ transitions with a lifetime-branching-ratio approach and by critically evaluating and assembling all recent literature data.

We have arrived at a set of $\boldsymbol{A}$ values for the important $4 s-p$ and $4 s-5 p$ transition arrays which is consistent with all recent literature data and possesses internal consistency between the two arrays. One may extend this scale to other transitions of Ar I, utilizing the comprehensive tables of Ar I transition probabilities contained in the
NSRDS-NBS compilation ${ }^{58}$ of 1969. This material, which is based on emission data, should be slightly renormalized as follows: (i) All transitions with reference numbers " $5 n$ " (the work of Bues et al. ${ }^{16}$ ) and " $6 n$ " (Corliss and Shumaker ${ }^{9}$ ), with a renormalization factor of 0.96 , (ii) Transitions with reference number " $8 n$ " (Malone and Corcoran ${ }^{7}$ ), with a factor of 0.94 , and (iii) Transitions with reference number " $9 n$ " (Wiese $e t$ al. ${ }^{17}$ ), with a factor of 0.89 .

However, these data are generally not as well established and accurate (most are estimated to be accurate within $\pm 50 \%$, see Ref. 58) as those for the $4 s-4 p$ and $4 s-$ $5 p$ arrays and further measurements are very desirable.

Finally, we should emphasize that the recommended transition probabilities are, as usually defined, those for an isolated atom. In plasmas, the radiating atoms are often significantly affected by the electric microfields of the surrounding electrons and ions (as well as possible external fields), and the emitted lines are then appreciably shifted and broadened and exhibit extended line wings. Thus, for the consistent application of these transition probability data in such emission experiments, the line intensity measurements need to include the line-wing intensity contributions, which may be best accounted for by corrections with a plasma line-shape model. ${ }^{70}$

\section{ACKNOWLEDGMENTS}

Two of us (W.L.W. and J.W.B.) performed part of this work during stays at the Ruhr-University, Bochum, West Germany, and the Justus-Liebig University, Giessen, West Germany, respectively. We would like to thank our hosts, Professor H.-J. Kunze and Professor M. Winnewisser, for their hospitality and the Alexander-vonHumboldt Foundation for providing us this opportunity.
${ }^{1}$ H. W. Drawin, Z. Phys. 146, 295 (1956).

${ }^{2}$ W. E. Gericke, Z. Astrophys. 53, 68 (1961).

${ }^{3}$ C. H. Popenoe and J. B. Shumaker, Jr., J. Res. Natl. Bur. Stand. Sec. A 69, 495 (1965).

${ }^{4}$ J. Richter, Z. Astrophys. 61, 57 (1965).

5 J. F. Bott, Phys. Fluids 9, 1540 (1966).

${ }^{6}$ P. B. Coates and A. G. Gaydon, Proc. R. Soc. London, Ser. A 293, 452 (1966).

${ }^{7}$ B. S. Malone and W. H. Corcoran, J. Quant. Spectrosc. Radiat.
Transfer 6, 443 (1966).

${ }^{8}$ M. S. Frish, Opt. Spectrosc. 22, 8 (1967).

${ }^{9}$ C. H. Corliss and J. B. Shumaker, Jr., J. Res. Natl. Bur. Stand., Sec. A 71, 575 (1967).

${ }^{10}$ J. B. Shumaker, Jr., and C. H. Popenoe, J. Opt. Soc. Am. 57, 8 (1967).

${ }^{11}$ W. L. Wiese, in Proceedings of the Eighth International Conference on Phenomena in Ionized Gases (Springer, Vienna, 1967), p. 447. 
12 J. Chapelle, A. Sy, F. Cabannes, and J. Blandin, J. Quant. Spectrosc. Radiat. Transfer 8, 1201 (1968).

${ }^{13}$ S. V. Desai and W. H. Corcoran, J. Quant. Spectrosc. Radiat. Transfer 8, 1721 (1968).

${ }^{14}$ B. Wende, Z. Phys. 213, 341 (1968).

${ }^{15}$ L. Bober and R. S. Tankin, J. Quant. Spectrosc. Radiat. Transfer 9, 855 (1969).

${ }^{16}$ I. Bues, T. Haag, and J. Richter, Astron. Astrophys. 2, 249 (1969); Technical Report, Institut für Experimentalphysik, Kiel University, 1969 (unpublished).

${ }^{17}$ W. L. Wiese, J. M. Bridges, R. L. Kornblith, and D. E. Kelleher, J. Opt. Soc. Am. 59, 1206 (1969).

${ }^{18}$ T. Wujec, Acta Phys. Pol. 36, 269 (1969).

${ }^{19}$ R. A. Nodwell, J. Meyer, and T. Jacobson, J. Quant. Spectrosc. Radiat. Transfer 10, 335 (1970).

${ }^{20}$ D. van Houwelingen and A. A. Kruithof, J. Quant. Spectrosc. Radiat. Transfer 11, 1235 (1971).

${ }^{21}$ A. D. Stokes, J. Phys. D 4, 930 (1971).

${ }^{22}$ J. von Specht, Astron. Astrophys. 34, 363 (1964).

${ }^{23}$ H. Nubbemeyer, J. Quant. Spectrosc. Radiat. Transfer 16, 395 (1976).

${ }^{24}$ R. C. Preston, J. Phys. B 10, 1377 (1977).

${ }^{25} \mathrm{H}$. Nubbemeyer and B. Wende, in Proceedings of the Thirteenth International Conference on Phenomena in Ionized Gases (Physical Society of the German Democratic Republic, Berlin, 1977), p. 147.

${ }^{26}$ R. S. F. Chang and D. W. Setser, J. Chem. Phys. 69, 3885 (1978).

${ }^{27}$ P. Baessler and M. Kock, J. Phys. B 13, 1351 (1980).

${ }^{28}$ K. P. Nick, Ph.D. thesis, Kiel University, 1982.

${ }^{29}$ M. J. G. Borge and J. Campos, Physica C 119, 359 (1983).

${ }^{30}$ M. J. G. Borge and J. Campos, J. Quant. Spectrosc. Radiat. Transfer 30, 433 (1983).

${ }^{31}$ K. P. Nick and V. Helbig, Phys. Scr. T8, 100 (1984).

${ }^{32}$ D. L. Adams and W. Whaling, J. Opt. Soc. Am. 71, 1036 (1981).

${ }^{33}$ K. Musiol, D. W. Jones, and W. L. Wiese, J. Quant. Spectrosc. Radiat. Transfer 29, 321 (1983).

${ }^{34}$ D. W. Jones and W. L. Wiese Phys. Rev. A 39, 110 (1989).

${ }^{35}$ A. Hirabayashi, S. Okuda, Y. Nambu, and T. Fujimoto, Phys. Rev. A 35, 639 (1987).

36J. Z. Klose, J. Opt. Soc. Am. 57, 1242 (1967).

${ }^{37}$ J. Z. Klose, J. Opt. Soc. Am. 58, 1509 (1968).

${ }^{38}$ D. A. Landman, Phys. Rev. 173, 33 (1968).

${ }^{39}$ G. M. Lawrence, Phys. Rev. 175, 40 (1968).

${ }^{40}$ Ya. F. Verolainen and A. L. Osherovich, Opt. Spektrosk. 25, 466 (1968) [Opt. Spectrosc. (USSR) 25, 258 (1968)].

${ }^{41}$ J. P. Grandin, D. Lecler, and J. Margerise, C. R. Acad. Sci., Ser. B 272, 929 (1971).

${ }^{42}$ Yu. I. Malakhov and V. G. Potyomkin, Opt. Spektrosk. 32, 245 (1972) [Opt. Spectrosc. (USSR) 32, 129 (1972)].

${ }^{43}$ J. Campos and B. Zurro, An. Fis. 69, 299 (1973).

${ }^{44}$ P. Erman and I. Martinson, Phys. Scr. 8, 269 (1973).

45J. Galy, H. Brunet, A. Birot, and J. L. Artigue, C. R. Acad. Sci., Ser. B 276, 23 (1973).

${ }^{46}$ D. J. G. Irwin, A. E. Livingston, and J. A. Kernahan, Nucl.
Instrum. Methods 110, 111 (1973).

${ }^{47}$ M. Chenier and P. A. Muskowitz, J. Phys. (Paris) 35, 40 (1974).

${ }^{48}$ A. L. Osherovich, V. N. Ivanov, and V. N. Groshkov, Vestn. Leningr. Univ., Fiz., Khim. 4, 7 (1974).

${ }^{49}$ P. Erman and S. Huldt, Phys. Scr. 17, 473 (1978).

${ }^{50}$ S. A. Kazantsev, E. D. Mishchenko, P. K. Telbizov, and M. P. Chaika, Opt. Spektrosk. 45, 816 (1978) [Opt. Spectrosc. (USSR) 45, 711 (1978)].

${ }^{51}$ M. J. G. Borge and J. Campos, J. Quant. Spectrosc. Radiat. Transfer 24, 263 (1980).

${ }^{52}$ T. Fujimoto, C. Goto, Y. Uetani, and K. Fukuda, Phys. Scr. 28, 617 (1983).

${ }^{53}$ D. J. Chornay, G. C. King, and S. J. Buckman, J. Phys. B 17, 3173 (1984).

${ }^{54}$ S. A. Kazantsev and A. V. Subbotenko, Opt. Spektrosk. 55, 767 (1983) [Opt. Spectrosc. (USSR) 55, 458 (1983)].

${ }^{55} \mathrm{U}$. Quadfasel and V. Helbig (unpublished).

${ }^{56} \mathrm{U}$. Ralfs and V. Helbig (unpublished).

${ }^{57}$ Z. Stryla, H. Pobee, W. Schade, and V. Helbig (unpublished).

${ }^{58}$ W. L. Wiese, M. W. Smith, and B. M. Miles, Atomic Transition Probabilities, Natl. Stand. Ref. Data Ser., Natl. Bur. Stand. (U.S.) Circ No. NSRDS-NBS 22 (U.S. GPO, Washington, D.C., 1969), Vol. II.

${ }^{59} \mathrm{~W}$. L. Wiese, in The Physics of Ionized Gases, edited by G. Pichler (The Institute of Physics, Zagreb, Yugoslavia, 1983), p. 435.

${ }^{60}$ W. L. Wiese, J. Quant. Spectrosc. Radiat. Transfer 40, 421 (1988).

${ }^{61} \mathrm{~J}$. W. Brault, in Proceedings of the 1978 Joint Organization for Solar Observation Workshop, Firenze, 1978, edited by G. Godoli, G. Noci, and A. Righini [Oss. Mem. Arcetri 106, 33 (1979)].

${ }^{62}$ K. Danzmann and M. Kock, J. Phys. B 13, 2051 (1980).

${ }^{63}$ W. Schade and V. Helbig, Phys. Lett. A115, 39 (1986).

${ }^{64}$ R. A. Lilly, J. Opt. Soc. Am. 66, 245 (1976).

${ }^{65}$ R. H. Garstang and J. Van Blerkom, J. Opt. Soc. Am. 55, 1054 (1965).

${ }^{66}$ P. D. Johnston, Proc. Phys. Soc. London, Ser. A 92, 896 (1967).

${ }^{67}$ P. F. Gruzdev and A. V. Loginov, Opt. Spektrosk. 38, 411 (1975) [Opt. Spectrosc. 38, 234 (1975)].

${ }^{68}$ H. M. Meiners, J. Quant. Spectrosc. Radiat. Transfer 9, 1493 (1969).

${ }^{69}$ As was recognized early on (Ref. 23), an Ar II line at 430.065 $\mathrm{nm}$ is blended with the Ar I 430.010-nm line and may have an influence on this line. Results by J. B. Shumaker, Jr., and C. H. Popenoe [J. Opt. Soc. Am. B 59, 980 (1969)] indicate, however, that in LTE argon plasmas the Ar II line contributes less than $1 \%$ at temperatures below $14000 \mathrm{~K}$. Practically all emission studies on this line were carried out at lower temperatures.

${ }^{70}$ W. L. Wiese, in Plasma Diagnostic Techniques, edited by $\mathbf{R}$. H. Huddlestone and S. L. Leonard (Academic, New York, 1965), Chap. 6. 